\title{
Medium chain acyl-CoA dehydrogenase deficiency: Human genome epidemiology review
}

\author{
Sophia S. Wang, $P h D^{1,2}$, Paul M. Fernhoff, $M D^{3}$, W. Harry Hannon, $P h D^{4}$, and Muin J. Khoury, MD, $P h D^{2}$
}

Medium chain acyl-CoA dehydrogenase (MCAD) is a tetrameric flavoprotein essential for the $\beta$-oxidation of medium chain fatty acids. MCAD deficiency (MCADD) is an inherited error of fatty acid metabolism. The gene for MCAD is located on chromosome one (1p31). One variant of the MCAD gene, G985A, a point mutation causing a change from lysine to glutamate at position 304 (K304E) in the mature MCAD protein, has been found in $90 \%$ of the alleles in MCADD patients identified retrospectively. There is a high frequency of MCADD among people of Northern European descent, which is believed to be due to a founder effect. MCADD is inherited in an autosomal recessive manner. Of patients clinically diagnosed with MCADD, $81 \%$ who have been identified retrospectively are homozygous for $\mathrm{K} 304 \mathrm{E}$, and $18 \%$ are compound heterozygotes for $\mathrm{K} 304 \mathrm{E}$. Clinical data on the probability of clinical disease indicates that MCADD patients are at risk for the following outcomes: hypoglycemia, vomiting, lethargy, encephalopathy, respiratory arrest, hepatomegaly, seizures, apnea, cardiac arrest, coma, and sudden and unexpected death. Long-term outcomes include developmental and behavioral disability, chronic muscle weakness, failure to thrive, cerebral palsy, and attention deficit disorder (ADD). Differences in clinical disease specific to allelic variants have not been documented. Factors that may increase risk for disease onset or modify disease severity are age when the first episode occurred, fasting, and presence of infection. Acute attacks must be treated immediately with appropriate intravenous doses of glucose. For those diagnosed, long-term management of the disease includes preventing stress caused by fasting and maintaining a high-carbohydrate, reduced-fat diet, and carnitine supplementation. Hospitalization costs attributable to morbidity and mortality from MCADD are unknown; MCADD is not a diagnosis in the International Classification of Disease, 10th Revision (ICD-10) codebook. Furthermore, the penetrance of the MCAD genotypes is unknown; there appears to be a substantial number of asymptomatic MCADD individuals and some uncertainty regarding which individuals will manifest symptoms and which individuals will remain asymptomatic. Several technologies are available to detect MCADD. Diagnostic technologies include DNA-based tests for K304E mutations using the polymerase chain reaction (PCR), and the detection of abnormal metabolites in urine. Screening technologies include tandem mass spectrometry (MS/MS), which detects abnormal metabolites mostly in blood. State programs are beginning to offer screening in newborns for MCADD using MS/MS. In addition, a private company currently offers voluntary supplemental newborn screening for MCADD to birthing centers. Genetics in Medicine, 1999:1(7):332-339.

Key Words: Medium-chain acyl-coenzyme A dehydrogenase, MCAD deficiency, epidemiology, review, gene

\section{GENE}

The medium chain acyl-CoA dehydrogenase (MCAD) gene is located at chromosome $1 \mathrm{p} 31$. The protein formed, MCAD, is

From 'Epidenic Intelligence Service, Invision of Appliot Public Hedllh Truming, Epitimi ology' Program Office. Centers for Discase Control and Preventim, the 'Cemters for Discasi

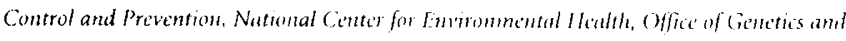
Disease Prevention; 'Enory University. Department of Pediatrics, Diveston of Medical (ie'-

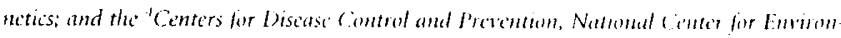

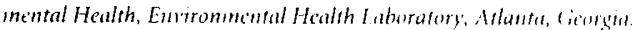

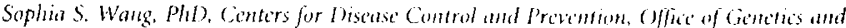
Discase Prevention, 4770) Buford Highwal, NE, MS K.28, Allutu, (id 31) 3.11-3724.

Received: Septomber 13, 1999 .

Accepted: Novernber 18, 1999 . a homotetramer and an oxidoreductase enzyme that catalyzes the first step of the medium-chain fatty acid oxidation cycle, a two-electron oxidation of fatty acyl-CoA thiolesters. MCAD is part of the acyl-CoA dehydrogenase (flavoprotein) family and is one of five acyl-CoA dehydrogenases that catalyze the $\beta$-oxidation of fatty acids.

\section{GENE VARIANTS}

Twenty six MCAD gene variants have been reported, ${ }^{1}$ with nine alleles of special interest listed in OMIM. ${ }^{2}$ This review will focus on K304E, the MCAD mutation reportedly found in $90 \%$ of all retrospectively identified MCAD deficient patients' alleles, and for whom $81 \%$ of all MCAD patients are homozy- 
gous; $18 \%$ of MCAD patients are compound heterozygous for K304E. Caucasian individuals of Northern European descent exhibit the highest frequency of MCADD genotypes. The carrier frequency of $\mathrm{K} 304 \mathrm{E}$ among this group is estimated to be $1: 40$ to $1: 100$ and the homozygote frequency is $1 / 6,500-$ $20,000 .^{3}$ Haplotype analyses have suggested that the K304E $\mathrm{MCAD}$ mutation is a founder effect from a single person in a Germanic tribe. ${ }^{4}$

Table 1 lists the frequencies of the $\mathrm{K} 304 \mathrm{E}$ mutation for the MCAD gene by country. Only two studies have been conducted on a population-based prospective cohort of newborns; most others are retrospective studies. Although heterozygotes have been identified with this method, few homozygotes have been identified; the frequencies of homozygosity shown in Table 1 are therefore mostly estimates based on the frequency of heterozygosity and using Hardy-Weinberg equilibrium. Despite this limitation, Ziadeh and colleagues' 1995 populationbased study of U.S. newborns demonstrated a frequency of homozygosity $(1 / 20,408)$ that is similar to those estimated in some northern European countries. ${ }^{5}$ Ziadeh and colleagues studied the first 80,371 newborns from a supplemental newborn screening program in Pennsylvania and eastern Ohio for more than 35 metabolic disorders. ${ }^{5}$ Despite the heterogeneity of the population, which included high proportions of Eastern

Table 1

Frequency by country of homozygosity and heterozygosity for K304E MCAD mutation

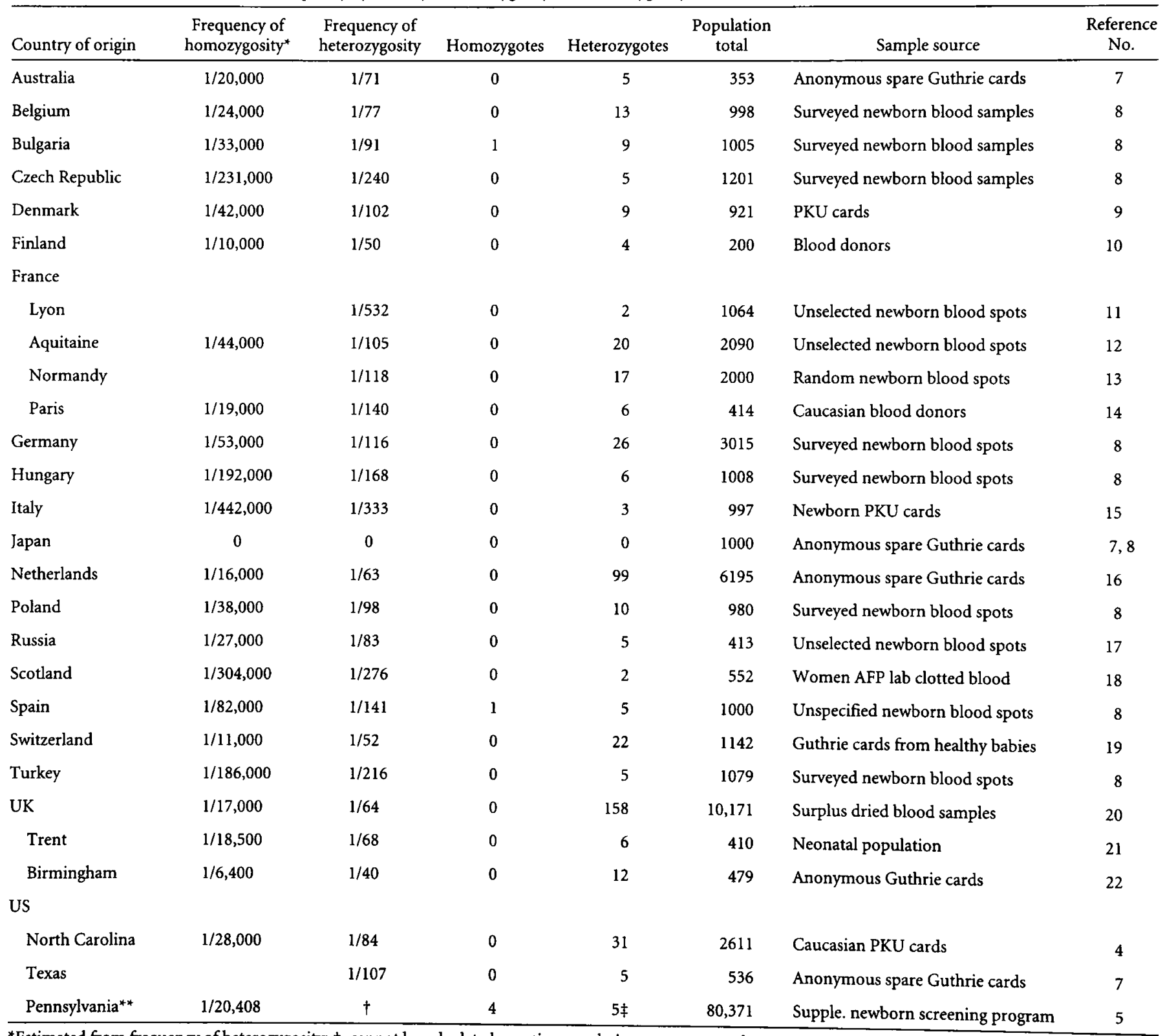

${ }^{*}$ Estimated from frequency of heterozygosity; $\uparrow$, cannot be calculated-entire population not genotyped; $\neq$, compound heterozygotes, not including carriers.
$* *$ Prospective population-based study. 
Europeans and Blacks, nine MCADD newborns were diagnosed from the first 80,371 newborns screened between November 1, 1992 and September 30, 1994 by tandem mass spectrometry. However, upon DNA sequencing of the nine MCADD newborns, $44 \%$ ( 4 of 9 ) were homozygous for K304E and $56 \%$ (5 of 9) were compound heterozygotes, versus the $81 \%$ homozygous and $18 \%$ compound heterozygotes previously calculated based on retrospective data. In the U.K., however, Pollitt and Leonard's 1998 prospective study of MCADD through the British Pediatric Surveillance Unit identified 57 people in England with MCADD, for an MCADD incidence of $4.5 / 100,000$. Of the 45 people with MCADD whose DNA has been analyzed thus far, 36 of $45(80 \%)$ were homozygous for $\mathrm{K} 304 \mathrm{E}$, and 9 of $45(20 \%)$ were heterozygous for K304E, ${ }^{6}$ which is consistent with data from retrospective studies (because data are still pending, this study is not listed in Table 1). It is important to note that most of the retrospective studies are conducted using spare blood samples and their results are not necessarily representative of the population, nor can they accurately assess incidence of the disorder. Only from population-based prospective cohort studies can incidence of a disorder be accurately assessed.

Research on MCADD is in its infancy, with most of the published articles having concentrated on the biochemical and molecular aspects of the disease. The natural history and longterm outcomes of patients with the disorder are still not clearly defined, nor is determining who will present with disease and who will remain asymptomatic. Population and epidemiologic information gathered from pilot and supplemental testing programs currently being conducted will begin to provide much needed information about the long-term clinical spectrum of the disorder.

\section{MCAD DEFICIENCY}

The first MCADD patients were identified in 1982, and between 1982 and 1994, more than 200 MCADD patients were identified. ${ }^{23} \mathrm{MCADD}$ is an autosomal recessive genetic disorder that leads to an abnormal protein product and subsequent inefficient enzymatic activity to metabolize medium chain fatty acids. The K304E MCAD mutation occurs when glutamate is substituted for lysine at position 304 in the mature MCAD protein. Individuals who are homozygous or compound heterozygous for an MCAD mutation may exhibit some clinical manifestations of MCADD. Differences in clinical manifestations specific to allelic variants have not yet been documented.

The incidence of MCADD can only be calculated from prospective population-based studies. Updated data from Ziadeh and colleagues' study in Pennsylvania and Eastern Ohio population have identified 16 newborns with MCADD out of 211,067 tested, for an incidence rate of $1 / 13,192 .{ }^{24}$ These rates are higher than the MCADD incidence rate found in Pollitt and Leonard's 1998 prospective study $(1 / 22,222)$ in the U.K. ${ }^{6}$

Although the frequency of the allele is rather high in Caucasian populations, based on rates of heterozygosity and under
Hardy-Weinberg conditions, there is a greater number of expected MCADD cases than are currently diagnosed. On the basis of the heterozygote frequency in New South Wales, Wilcken and colleagues (1994) estimated that the MCADD incidence rate should be $1 / 15,000$ to $1 / 20,000$ or 42 cases in their 1975-1984 study. ${ }^{25}$ However, only 9 MCADD individuals ( $21 \%$ of expected) were identified, and only 7 of an expected 31 from 1985-1991 were identified. Similarly, Fromenty and colleagues (1996) found that of 414 French blood donors, the mutant gene frequency of $1 / 138$ produces a homozygote frequency of $1 / 19,000$ according to Hardy-Weinberg, for an expected 42 MCADD individuals. ${ }^{14}$ However, only six cases of MCADD were reported.

The numbers of MCADD cases observed have been consistently lower than the number expected. Two hypotheses have been offered to explain this lower than expected number of cases. First, symptomatic newborns may be misdiagnosed as having SIDS or Reye's syndrome or may die before MCADD is detected. Second, a substantial number of people with MCADD may be asymptomatic and unidentified, suggesting a penetrance of $<100 \%$. Although misdiagnosis and early deaths may account for some unidentified cases, they are not likely to account for all of them.

There is a wide spectrum of disease severity ranging from no symptoms to sudden death. The following two sections summarize the disease outcomes associated with MCADD, and the risk factors for presenting with the various outcomes. Genotypic-phenotypic correlations have not been documented. Clinical manifestations presented in the following sections are therefore general outcomes of the disorder and not specific to any allelic variant.

\section{DISEASE ASSOCIATIONS}

\section{Clinical symptoms and outcomes}

Clinical signs and symptoms usually first occur during a child's first 3 years of life (average is 1 year). Neonatal onset is possible, but presentation rarely occurs in adults. ${ }^{26} \mathrm{MCADD}$ is an episodic disorder. Children suddenly present with a constellation of features; the most common features include vomiting and lethargy. Hypoglycemia, encephalopathy, respiratory arrest, hepatomegaly, seizures, coma, apnea, cardiac arrest, and sudden death have also been documented. Long-term outcomes may include developmental and/or behavioral disabilities including mental retardation, cerebral palsy, and ADD. Table 2 displays results from three studies that have documented symptoms observed in clinically diagnosed MCADD patients. In summary, although the signs and symptoms of MCADD are variable, they are similar to those seen in other encephalopathies: vomiting, lethargy, seizures, and progressive coma. Routine clinical laboratory findings include hypoketotic hypoglycemia, with hyperammonemia and abnormal liver function. Although these features are presented in this review individually, clinical presentation consists of any combination of these features. 
Table 2

Presenting symptoms observed in MCADD patients

\begin{tabular}{lccc}
\hline Clinical symptoms & 120 cases (27) & 65 cases (28) & First 23 cases (29) \\
\hline Hypoglycemia & n.r. & $96 \%$ & $91 \%$ \\
Probable infection & $85 \%$ & $74 \%$ & n.r. \\
Lethargy & $84 \%$ & $100 \%$ & $26 \%$ \\
Vomiting & $66 \%$ & $60 \%$ & $52 \%$ \\
Coma/encephalopathy & $49 \%$ & n.r. & $78 \%$ \\
Respiratory arrest & $48 \%$ & n.r. & $4 \%$ \\
Hepatomegaly & $44 \%$ & $59 \%$ & $30 \%$ \\
Seizures & $43 \%$ & $29 \%$ & $17 \%$ \\
Apnea & $37 \%$ & n.r. & n.r. \\
Cardiac arrest & $36 \%$ & n.r. & n.r. \\
Sudden death & $18 \%$ & $26 \%$ & $17 \%$ \\
\hline
\end{tabular}

n.r., not reported.

Numerous case reports and case series have documented clinical outcomes for people with MCADD who were untreated prior to presentation. Few studies, however, have reviewed the outcomes of MCADD individuals beyond the scope of the case reports. Systematic evaluations of morbidity and mortality in population-based studies have not been conducted. Furthermore, because MCADD is not listed in the ICD-10 or previous codebooks, estimates of morbidity, hospitalization burden, and annual costs of the disease cannot be calculated. Two studies that did attempt to quantify the number of people affected or presenting with various clinical outcomes are summarized in Table 2. To date, Iafolla and colleagues (1994) have reviewed the largest number of MCADD cases. All 120 patients in the study were of European descent: 112 from the United States, and the remaining 8 from the United Kingdom, Australia, Canada, or Ireland. ${ }^{27}$ These cases were clinically identified through physicians and subsequently analyzed and confirmed by at least two methods of MS. Touma and Charpentier (1992) reviewed outcomes for 65 MCADD patients identified upon acute onset of the disease at hospitals who had their MCADD diagnosis confirmed by GC/MS. ${ }^{28} \mathrm{~A}$ third study summarized the outcomes of the first 23 reported cases of MCADD in the world (mostly from Europe), which were compiled from published case reports of each individual. ${ }^{29}$ The prevalence of clinical symptoms reported for patients in all three studies are summarized in Table 2. It is important to note that there is overlap between the studies referenced in Table 2. For example, some of the patients reported by Roe and Coates $(1989)^{23}$ contribute to Iafolla and colleagues'27 patient population, which also includes some patients reported by Touma and Charpentier, ${ }^{28}$ which in turn includes some patients from Vianey Liaud and colleagues ${ }^{29} 1987$ study. However, because it is unknown how many or which patients are reported in duplicate among these studies, the studies are presented separately for the purposes of this review. Therefore, differences and similarities between the outcomes reported for each of these studies should be compared with the knowledge that there is some duplicate reporting among the studies.

As shown in Table 2, hypoglycemia is the most common finding, present in over $90 \%$ of MCADD patients, followed by lethargy and vomiting. Most children with MCADD present with lethargy after a period of fasting, and more than one-half of MCADD patients exhibited vomiting. The study of Iafolla and colleagues was the only study to report encephalopathy. These findings are supported by the most recent prospective study on MCADD 6 in the U.K., in which 37 of 46 MCADD patients $(80 \%)$ suffered from acute hypoglycemia, and 23 of 46 (50\%) experienced encephalopathy.

The presence of seizures ranges from $17-43 \%$ among the three studies. Despite the overwhelming incidence of coma in the first 23 MCADD patients reported (78\%), coma was not assessed in the later studies; reasons for this include the possible reclassification as encephalopathy in later studies, or a possible decrease in coma due to interventions preventing coma associated with the increased awareness of MCADD.

\section{Sudden death}

Two of nine (22\%) MCADD patients identified through newborn screening in Pennsylvania died suddenly and unexpectedly. ${ }^{5}$ In a study by the British Pediatric Association Surveillance System, 10 of $46(21 \%)$ MCADD patients who presented acutely, died. ${ }^{6}$

Case studies have often documented sudden deaths among MCADD patients. The mean age of death among children with MCADD was 18.5 months. Among MCADD cases documented to date, patients have a $20-25 \%$ mortality rate at first presentation. ${ }^{27,30}$ However, these percentages may be lower if a number of MCADD patients remain undiagnosed during their lifetime. Furthermore, increased clinical awareness of fatty acid oxidation disorders in general may have significantly changed the observed outcomes over recent years.

Although various studies have investigated the relationship between sudden death and MCADD, few have strictly investigated SIDS (defined as sudden and unexpected death among infants less than 1 year of age) and MCADD. These studies have varied in quality, and the results have been inconsistent. Furthermore, MCADD cases reported to date involve patients who are symptomatic even though many people with MCADD will be asymptomatic. Because the prevalence of symptoms is a risk factor for death, the relatively high incidence of death in clinically reported MCADD patients is not unexpected.

Table 3 lists the estimated K304E carrier frequency in SIDS populations. These studies demonstrate that the frequency of this mutation in SIDS populations is similar to that in general newborn populations. These results indicate that this most common MCADD mutation has not accounted for much of the SIDS incidence in populations studied to date..$^{12,13}$ Thus, universal newborn screening would not be expected to significantly lower rates of SIDS. However, given the use of convenient samples (not population-based) and small sample sizes, a large variance in allelic frequencies is seen. Nevertheless, al- 
Table 3

Frequency of the K304E MCAD allelic variant among SIDS patients

\begin{tabular}{|c|c|c|c|c|c|}
\hline $\begin{array}{l}\text { No. of } \\
\text { SIDS }\end{array}$ & Population & $\begin{array}{c}\text { K304E } \\
\text { homozygotes }\end{array}$ & $\begin{array}{c}\mathrm{K} 304 \mathrm{E} \\
\text { heterozygotes }\end{array}$ & $\begin{array}{l}\text { Heterozygote } \\
\text { frequency }\end{array}$ & $\begin{array}{l}\text { Reference } \\
\text { No. }\end{array}$ \\
\hline 309 & US-Maryland & 1 & 1 & $1 / 309$ & 31 \\
\hline 100 & France-Aquitaine & 0 & 1 & $1 / 100$ & 12 \\
\hline 1224 & US-California & 0 & 3 & $1 / 375$ & 32 \\
\hline 120 & Denmark-Copenhagen & 0 & 1 & $1 / 120$ & 33 \\
\hline 233 & W. Scotland & 0 & 3 & $1 / 78$ & 16 \\
\hline 262 & US-Maryland & 0 & 3 & $1 / 88$ & 34 \\
\hline 67 & US-New York & 0 & 3 & $1 / 22$ & 35 \\
\hline 119 & US-North Carolina & 0 & 4 & $1 / 30$ & 36 \\
\hline 153 & Germany & 0 & 0 & & 37 \\
\hline 708 & Australia & 0 & 7 & $1 / 101$ & 38 \\
\hline
\end{tabular}

though MCADD may not account for much of SIDS, it is still clearly a cause of sudden death in young children.

\section{Long-term outcomes}

Iafolla and colleagues have conducted the only long-term outcome evaluation of MCADD patients; their results are summarized in Table 4. ${ }^{27}$ Developmental, behavioral, and neurological disabilities are beginning to be documented. Iafolla and colleagues ${ }^{27}$ followed 73 MCADD survivors older than 2 years of age; they found that $32 \%$ had abnormal results on formal psychodevelopmental tests, including speech and language delay and behavioral problems. Clinical onset between 12 and 18 months of age, which included seizures or encephalopathy, correlated strongly with the development of speech disability. ${ }^{27} \mathrm{~A}$ smaller study with longer follow-up reported handicaps and failure in school in MCADD patients. ${ }^{25}$

Table 4

Long-term consequences in 97 surviving MCADD patients 6 months to 9 years old*

\begin{tabular}{lc}
\hline Long-term outcome & $\%$ of MCADD patients \\
\hline Developmental disability** & $16 \%$ \\
Speech and language delay** & $22 \%$ \\
Behavioral problems $^{* *}$ & $15 \%$ \\
At least one of the above outcomes & $32 \%$ \\
Attention deficit disorder** & $12 \%$ \\
Proximal muscle weakness & $16 \%$ \\
Chronic seizure disorder & $14 \%$ \\
Cerebral palsy & $9 \%$ \\
Failure to thrive & $10 \%$ \\
Aphasia following illness & $5 \%$ \\
\hline${ }^{*}$ See reference 27. & \\
${ }^{*}$ Out of 73 patients for whom psychodevelopmental information was avail- \\
able.
\end{tabular}

In Pollit and Leonard's 1998 study, conducted with the British Pediatric Association Surveillance System, 6 of 36 surviving MCADD patients suffered neurological damage. Because follow-up has not been conducted past childhood or past the immediate post-illness period, it is unknown whether this number would increase if follow-up were performed through adulthood. ${ }^{39}$ Neurological defects among MCADD patients have also been documented in Denmark. ${ }^{40}$

Other long-term outcomes of MCADD include episodic hypoglycemia and muscle weakness. Hypoglycemia can be induced by several measures such as illness or immunization. Treatment for hypoglycemia has been required for MCADD patients receiving the diphtheria-pertussis-tetanus vaccine, and for children with chicken-pox and otitis media. Risk factors for muscle weakness include an older age at diagnosis $(>3$ years of age), increased number of clinical events and hospitalizations before diagnosis, and time between clinical onset and diagnosis with longer delays increasing risk. ${ }^{27}$

Failure to thrive, seizure disorders, cerebral palsy, and ADD have also been reported as long-term outcomes among MCADD patients. A strong correlation existed between seizure at onset and cerebral palsy. Patients with ADD were more likely to have had seizures, encephalopathy, and hyperammonemia at the time of onset, as well as more episodes of clinical illness before and after diagnosis. MCADD patients diagnosed with ADD were also older at diagnosis of MCADD than patients who did not have ADD. The percentage of ADD cases attributable to MCADD is not known at present but is probably small.

\section{INTERACTIONS WITH RISK FACTORS}

MCADD results in only partial blockage of fatty acid oxidation; patients are therefore still able to metabolize shortchained fatty acids. As a result, a stimulus is needed for clinical symptoms to present. It is often in times of metabolic stress induced by fasting or infection when the demands on fatty acid 
oxidation are particularly high, that an MCADD patient may present with symptoms.

Factors that affect clinical outcomes include prolonged fasting, infections or recent immunization, the patient's age when the first episode occurred, and a family history of SIDS or a sibling diagnosed with MCADD. In other words, the phenotype of MCADD is dependent on the degree of metabolic stress and a combination of other factors such as fever, infections, etc. ${ }^{1}$

\section{Fasting}

Episodes of hypoketotic hypoglycemia, vomiting, lethargy, seizures, and coma often are precipitated by fasting. Procedures such as anesthesia and surgery, which are preceded by fasting, can therefore also precipitate episodic events. Fasting leads to hypoglycemia, then vomiting or lethargy. Seizures and coma occur in fewer but more severe cases. The specific magnitude of risk associated with fasting has not yet been assessed or calculated.

\section{Infection/intercurrent illness}

Hypoglycemia requiring hospitalization is the most common outcome resulting from infection. This is often due to the vomiting and/or diarrhea brought on by the illness. Therefore, episodes of hypoketotic hypoglycemia, vomiting, lethargy, seizures, and coma all occur in association with infection. If these episodes are severe, death can result. Three of 22 MCADD patients with chicken pox required treatment for hypoglycemia during an illness. ${ }^{27}$ One of two MCADD newborns who died in the supplemental testing program in Pennsylvania died during intercurrent illness. ${ }^{5}$ Furthermore, physiologic stresses due to intercurrent infections frequently lead to the loss of appetite in children who are ill, resulting in a period of fasting and subsequent metabolic imbalance and induction of episodes.

\section{Immunizations}

Four percent of MCADD patients suffered from hypoglycemia requiring hospitalization after being immunized. ${ }^{27}$ One of the two MCADD patients who died after being identified through the supplemental newborn testing program provided in Pennsylvania, died after a metabolic crisis stimulated by immunization. ${ }^{5}$

\section{Age of first episode}

Disease severity varies with a patient's age of first presentation. Episodes usually occur when children are between 3 months and 3 years of age, with an average of 12 months. The risk of dying from MCADD is higher among patients whose first episode occurs after the age of 1 year. If the first episode occurs when the child is less than 1 year of age, the outcome usually consists of hypoglycemia and is not fatal. However, if the first episode doesn't occur until the child is older than 1 year of age, significant mortality can result. The highest risk for death occurs among children whose symptoms first appear when they are 15 to 26 months old. ${ }^{26}$ Roe and Coates ${ }^{23}$ re- ported that clinical onset between 12 and 18 months was usually marked by seizures or encephalopathy, and correlated strongly with the development of a speech disability. Twelve of $63(19 \%)$ infants less than 1 year old died during their first MCADD episode. Nine of 41 (22\%) children older than 1 year died during their first episode. ${ }^{26}$

\section{Age of diagnosis}

The older a child is when the MCADD diagnosis is made, the higher the child's risk for muscle weakness and ADD. ${ }^{27}$ Risk for muscle weakness also increases as the time between clinical onset and diagnosis increases and the number of clinical events before diagnosis increases. Whether age is a true risk factor or simply a surrogate for delay in diagnosis needs to be explored.

\section{Seizures}

MCADD patients who present with seizures are at an increased risk for death, recurrent seizures, or cerebral palsy in the future. ${ }^{27}$

\section{Family history}

Family history of MCADD is technically not a risk factor for having clinical symptoms; however, the association between the two is consistent with the autosomal recessive inheritance of the disease. A proportion of MCADD patients in all studies have siblings who are affected with MCADD or have siblings who died unexpectedly and suddenly, with some deaths diagnosed as SIDS. In one-half of the families studied by Touma and Charpentier, ${ }^{28}$ patients had one or more siblings who died prior to the proband's presentation. Of 43 families identified by Roe and Coates, ${ }^{26}$ one-third had one or more unexplained sibling death. ${ }^{26}$ Iafolla and colleagues' 1994 study $^{27}$ of 120 MCADD patients reported that $32 \%$ of patients had siblings who were either known to have MCADD or had died of SIDS. Of 57 cases collected prospectively by the British Pediatric Association Surveillance System between 1994 and 1996, five had siblings who died. Iafolla and colleagues reported that $20 \%$ of families (19 of 94) had one or more unexplained sibling death. ${ }^{41}$ Similarly, of 109 siblings of MCADD children, eight had died from MCADD. ${ }^{23}$

To date an average of $30 \%(10-50 \%)$ of MCADD patients have affected siblings. The presence of an affected sibling may thus be a risk factor for MCADD; it is also possible for the association to be due to ascertainment bias. Roe and Coates ${ }^{23}$ concluded that younger siblings of children with MCADD were the ones most at risk for a fatal first episode. However, the percentage of specific clinical outcomes among MCADD patients with affected siblings has not been determined. Studies comparing MCADD patients with family history of MCADD to MCADD patients identified in the population have not been conducted. Therefore, whether MCADD patients with affected siblings have a higher incidence of clinical symptoms than patients identified in the population is unknown. In other words, we do not know whether the penetrance of MCADD for clinical outcomes is higher among patients with a family history of 
the disorder than among patients identified in the general population.

\section{LABORATORY TESTS}

The main technologies available for detecting individuals with MCADD involve the detection of abnormal biochemical markers in the blood or urine. Tandem mass spectrometry (MS/MS) detects abnormal metabolites mainly in blood samples. Although MS/MS is used as an initial screening modality, confirmation of MCADD diagnosis is conducted with urine organic acid profile and/or DNA mutation analysis. Dicarboxylic aciduria is an important diagnostic element and detection of urinary acylglycines (hexanoylglycine, phenylpropionylglycine, and suberylglycine) are traditional and accurate diagnostic methods for MCADD. Confirmation of MCADD through blood samples and dried-blood spots can be conducted with DNA/PCR technology.42

MS/MS is the most recent technology developed and the most suitable for large-scale population screening for MCADD. MS/MS detects octanoylcarnitine and other acylcarnitines in blood and is currently the method used in a supplemental screening program offered by a private company that screens most newborns in Pennsylvania and eastern Ohio. ${ }^{43}$ MS/MS is also the method of choice for North Carolina, California, and Massachusetts in their expanded newborn screening programs that will include screening for MCADD. MS/MS screening is being piloted in the U.K. and Australia.

\section{POPULATION TESTING}

North Carolina and Massachusetts are currently testing for MCADD as part of their newborn screening program. Soon, California will offer optional testing for MCADD to parents. In addition, Neo Gen Screening (Pittsburgh, PA) offers voluntary MCADD testing to newborns born at birthing centers in the Northeast; $75 \%$ of Pennsylvania's newborns receive this test in addition to the testing provided by the Pennsylvania state screening program. ${ }^{21}$ In Europe, the Institute of Child Health in London is planning a population-based pilot study of MS/MS screening on newborns, which includes screening for MCADD.

According to the 1997 British Health Technology Assessment report, MCADD satisfies most criteria for universal newborn screening: its incidence in relevant populations is known, it is associated with significant morbidity or mortality, effective treatment is available, there is a period before onset of symptoms during which intervention improves outcome, and MS/MS allows safe and simple testing for the disease on the routine newborn screening blood spots. Data from population studies and screening programs currently being conducted will be useful in assessing the long-term outcomes and effectiveness of screening for MCADD.

\section{References}

1. Andresen B, Bross P, Jensen T, Knudsen I, Winter V, Kolvraa S, Bolund L, Gregersen N. Molecular diagnosis and characterization of medium-chain acyl-CoA dehydrogenase deficiency. Scand J Clin Lab Invest 1995;220:9-26.

2. OMIM (Online Mendelian Inheritance in Man) Baltimore. Johns Hopkins University, Center for Medical Genetics, 1998. http://www3.ncbi.nlm.nih.gov/htbin-post/ Omim/dispmim?201450

3. Seymour CA, Thomason MJ, Chalmers RA, Addison GM, Bain MD, Cockburn F, Littlejohns P, Lord J, Wilcox AH. Newborn screening for inborn errors of metabolism: A systematic review. Health Technol Assess 1997;1:1-95.

4. Gregersen N, Winter V, Curtis D, Deufel T, Mack M, Hendrickx J, Willems PJ, Ponzone A, Parrella T, Ponzone R, Parrella T, Ponzone R, Ding I, Zhang W, Chen Y, Kahler S, Roe C, Kolvraa S, Schneiderman K, Andresen B, Bross P, Bolund L. Medium-chain acyl-CoA dehydrogenase (MCAD) deficiency: The prevalent mutation $\mathrm{G} 985$ (K304E) is subject to a strong founder effect from Northwestern Europe. Hum Hered 1993;43:342-350

5. Ziadeh R, Hoffman E, Finegold D, Hoop RC, Brackett JC, Strauss AW, Naylor EW. Medium chain acyl-CoA dehydrogenase deficiency in Pennsylvania: Neonatal screening shows high incidence and unexpected mutation frequencies. Pediatr Res 1995;37:675-678

6. Pollitt RJ, Leonard JV. Prospective surveillance study of medium chain acyl-CoA dehydrogenase deficiency in the UK. Arch Dis Child 1998;79:116-119.

7. Matsubara Y, Narisawa K, Tada K, Ikeda H, Yeqi Y, Danks DM, Green A, McCabe ER. Prevalence of $\mathrm{K} 329 \mathrm{E}$ mutation in medium-chain acyl-CoA dehydrogenase gene determined from Guthrie cards. Lancet 1991;338:552-553.

8. Tanaka K, Gregersen N, Ribes A, Kim J, Kolvraa S, Winter V, Eiberg H, Martinez G, Deufel T, Leifert B, Santer R, Francois B, Pronicka E, Laszlo A, Kmoch S, Kremensky I, Kalaydjicva L, Ozalp I, Ito M. A survey of the newborn populations in Belgium, Germany, Poland, Czech Republic, Hungary, Bulgaria, Spain, Turkey, and Japan for the G985A variant allele with haplotype analysis at the medium chain acyl-CoA dehydrogenase gene locus: clinical and evolutionary consideration. Pediatr Res 1997;41:201-209.

9. Gregersen N, Winter V, Kolvraa S, Andresen B, Bross P, Blakemore A, Curtis D, Bolund $L$. Molecular analysis of medium-chain acyl-CoA dehydrogenase deficiency: A diagnostic approach. In: Coates P, Tanaka K, editors. New Development in Fatty Acid Oxidation. NY: Wiley-Liss, 1992:441-452.

10. Schwarz EI, Skobeleva NA, Ilonen J, Akerblom HK. The frequency of MCAD mutation (K329E) in the Finnish population (Abstract). Eur J Pediatr 1995;154:501A.

11. Vianey-Saban C, Dorche C, Divry P, Divry P, Lahet C, Mathieu M. Screening of the A 985 to $G$ mutation of the medium chain acyl-CoA dehydrogenase gene in RhoneAlpes area in a pilot study. In: Farriaux JP, Dhondt JL, editors. New Horizons in Neonatal Screening. Amsterdam: Elsevier Science B.V., 1994:257-259.

12. Ged C, El Sebai H, de Verneuil H, Parrot-Rouleau F. Is genotyping useful for the screening of medium chain acyl-CoA dehydrogenase deficiency in France? J Inherit Metab Dis 1995;18:253-256.

13. Lecoq I, Mallet E, Bonte J, Travert $\mathrm{G}$. The A985 to $\mathrm{G}$ mutation in the medium chain acyl-CoA dehydrogenase gene and sudden infant death syndrome in Normandy. Acta Paediatr 1996;85:145-147.

14. Fromenty B, Mansouri A, Bonnefont J. Most cases of medium-chain acyl-CoA dehydrogenase deficiency escape detection in France. Hum Genet 1996;97:367-368.

15. Cavalli-Sforza L, Menozzi P, Piazza A. The History and Geography of Human Genes. Princeton, NJ: Princeton University Press, 1995.

16. de Vries H, Niezen-Koning K, Kliphuis Kliphuis JW, Smit GP, Scheffer H, ten Kate LP. Prevalence of carriers of the most common medium-chain acyl-CoA dehydrogenase (MCAD) deficiency mutation (G985A) in The Netherlands. Hum Genet 1996;98:1-2.

17. Levin M, Zhang $Y$, Adams V, Schwartz E, McCabe E. MCAD K329E mutant allele frequency in Russia: unselected sampling with newborn screening specimens and need for automation (Abstract). Am J Hum Genet 1992;51:A172.

18. Dundar M, Lanyon W, Conner J. Scottish frequency of the common G985A mutation in the medium-chain acyl-COA (MCAD) gene and the role of MCAD deficiency in sudden infant death syndrome (SIDS). J Inherit Metab Dis 1993;16:991-993.

19. Conne B, Zufferey R, Belin D. The A985G mutation in the medium-chain acyl-CoA dehydrogenase gene: High prevalence in the Swiss population resident in Geneva. Inherit Metab Dis 1995;18:577-83.

20. Seddon H, Green A, Gray R, Leonard JV, Pollitt RJ. Regional variations in mediumchain acyl-CoA dehydrogenase deficiency. Lancet 1995;345:135-136.

21. Blakemore A, Singleton H, Pollitt R, Engel P, Kolvraa S, Gregersen N, Curtis D. Frequency of the G985A MCAD mutation in the general population. Lancet 1991; 337:298-299.

22. Matsubara Y, Narisawa K, Tada K. Medium-chain acyl-CoA dehydrogenase deficiency: Molecular aspects. Eur J Pediatr 1992;151:154-159.

23. Roe C, Coates P. Mitochondrial fatty acid oxidation disorders. Chap 45 In: Scriver C, 
Beaudet A, Sly W, Valle D, editors. The Metabolic Basis of Inherited Disease. New York: McGraw Hill, 1994;1501-1533.

24. Pollitt RJ, Green A, McCabe CJ, Booth A, Cooper NJ, Leonard JV, Nicholl J, Nicholson P, Tunaley JR, Virdi NK. Neonatal screening for inborn errors of metabolism: Cost, yield and outcome. Health Technol Assess 1997;1:1-202.

25. Wilcken B, Hammond J, Silink M. Morbidity and mortality in medium chain acy] coenzyme A dehydrogenase deficiency. Arch Dis Child 1994;70:410-412.

26. Roe C, Coates P. Acyl-CoA dehydrogenase deficiencies. Part 5, Chap. 33 In: Scriver C, Beaudet A, Sly W, Valle D, editors. The Metabolic Basis of Inherited Disease. New York: McGraw Hill, 1989;889-914.

27. Iafolla $A$, Thompson $R$, Roe $C$. Medium-chain acyl-coenzyme A dehydrogenase deficiency: Clinical course in 120 affected children. J Pediatr 1994;124:409-415.

28. Touma E, Charpentier C. Medium chain acyl-CoA dehydrogenase deficiency. Arch Dis Child 1992;67:142-145.

29. Vianey-Liaud C, Divry P, Gregersen N, Mathieu M. The inborn errors of mitochondrial fatty acid oxidation. J Inherit Metab Dis 1987;10:159-198.

30. Wilcken B, Carpenter K, Hammond J. Neonatal symptoms in medium chain acyl coenzyme A dehydrogenase deficiency. Arch Dis Child 1993;69:292-294.

31. Boles R, Buck E, Blitzer M, Platt MS, Cowan TM, Martin SK, Yoon H, Madsen JA, Reyes-Mugica M, Rinaldo P. Retrospective biochemical screening of fatty acid oxidation disorders in postmortem livers of 418 cases of sudden death in the first year of life. J Pediatr 1998;132:924-933.

32. Arens R, Gozal D, Jain K, Muscati S, Heuser ET, Williams JC, Keens TG, Ward SL. Prevalence of medium-chain acyl-coenzyme A dehydrogenase deficiency in the sudden infant death syndrome. J Pediatr 1993;122:715-718.

33. Lundemose J, Gregersen N, Kolvraa S, Norgaard Pedersen B, Gregersen M, HelwegLarsen $\mathrm{K}$, Simonsen $\mathrm{J}$. The frequency of disease-causing point mutation in the gene coding for medium-chain acyl-CoA dehydrogenase in sudden infant death syndrome. Acta Paediatr 1993;82:544-546.

34. Rindfleisch M, Gur S, Birtzer M, Cowan T, Chinsky J. Screening for MCAD deficiency in SIDS. Pediatr Res 1993;33:132A.

35. Chinsky J, Tolsma T, Cowan T, Blitzer M. Medium-chain acyl-CoA dehydrogenase (MCAD) deficiency and SIDS: An analysis of post-mortem liver samples for the presence of the common MCAD mutant allele. Am J Hum Genet 1991;49:A183.

36. Chen Y, Millington D, Zhang W, Ding J, Terada N, Iafolla A, Kahler S, Roe C. Workshop and Abstracts from the Second International Symposium on Clinical Biochemical and Molecular Aspects of Fatty Acid Oxidation Defects. Philadelphia, 1991:W-7.

37. Deufel T, Mack M, Muller B, Wiske J, Findeisen-Huls M, Bajanowski T, Jorch G, Brinkman B, Gregersen N, Roscher A. Workshop and Abstracts from the Second International Symposium on New Developments in Fatty Acid Oxidation; Philadelphia, 1991:P-10.

38. McGill J, Brown $\mathrm{N}$, Thomson $\mathrm{D}$, et al. Failure to identify medium-chain acyl-CoA dehydrogenase (MCAD) deficiency by mutation analysis in 708 infants who died from sudden infant death syndrome (Abstract). Australasian Inborn Errors of $\mathrm{Me}$ tabolism Conference, Bondi Beach. November, 1991.

39. Pollitt RJ, Leonard JV. Medium-chain acyl-CoA dehydrogenase deficiency. London: Surveillance Unit of the College of Paediatrics \& Child Health, Annual Report. 1996;25-26.

40. Andresen B, Bross P, Udvari S, Kirk J, Gray G, Kmoch S, Chamoles N, Knudsen I, Winter V, Wilcken B, Yokota I, Hart K, Packman S, Harpey JP, Saudubray JM, Hale DE, Bolund L, Kolvraa S, Gregersen N. The molecular basis of medium-chain acylCoA dehydrogenase (MCAD) deficiency in compound heterozygous patients: Is there correlation between genotype and phenotype? Hum Mol Genet 1997;6:695-707.

41. Iafolla A, Millington D, Chen X, Ding J, Kahler S, Roe C. Natural course of the medium chain acyl-CoA dehydrogenase deficiency. Am J Hum Gen 1991;49:99.

42. Iolascon A, Parrella T, Perrotta S, Guardamagna O, Coates PM, Sartore M, Surrey S,
Fortina P. Rapid detection of medium chain acyl-CoA dehydrogenase gene mutations by non-radioactive, single strand conformation polymorphism minigels. J Med Genet 1994;31:551-554.

43. Chace D, Hillman S, Van Hove J, Naylor EW. Rapid diagnosis of MCAD deficiency: Quantitative analysis of octanoylcarnitine and other acylcarnitines in newborn blood spots by tandem mass spectrometry. Clin Chem 1997;43:2106-2113.

\section{Appendix}

\section{Internet sites}

\section{General resources}

National Organization for Rare Disorders, Inc.: (http. //www. stepstn.com/nord/rdb sum/585.htm)

Fatty Acid Oxidation Disorder Network: (http. //www.cinternet.net/FOD/mcad3.html) and (http://www.cinternet.net/ $\mathrm{FOD} /$ mcad2.html)

\section{Genetic databases}

Gene Cards; (http. //bioinfo.weizmann.ac.il/cards-bin/ carddisp?ACADM\&search $=$ mcad\&suff $=t x t$ )

The Genome Database: (http. //gdbwww.gdb.org/gdb-bin/ genera/accno?GDB:118958)

Human Gene Mutation Database: (http.//www.uwcm.ac. $\mathrm{uk} / \mathrm{uwcm} / \mathrm{mg} / \mathrm{search} / 1$ 18958.html)

Online Mendelian Inheritance in Man: (http. //www3.ncbi. nlm.nih.gov/htbin-post/Omim/dispmim?201450)

\section{Educational resources}

University of California, San Diego: (http. //chem-faculty.ucsd.edu/harvey/MCAD/index.html)

Wittenberg University: (http. //blackboxl.wittenberg.edu/ academics/chem/faculty staff/aanderson/ chem100 Sum98/ssato/s01 ssato.htm)

University College London (http. //www.biochem.ucl.ac. $\mathrm{uk} / \mathrm{bsm} / \mathrm{pdbsum} / 3 \mathrm{mdd} / \mathrm{main} . \mathrm{html}$ )

\section{Support groups}

Fatty Oxidation Disorder Family Support Group (FOD): (http. //www.familyvillage.wisc.edu/lib mcad.htm)

United Mitochondrial Disease Foundation, Southern CA Support Group: (http. //biochemgen.ucsd.edu/umdf) 